\title{
Elastic properties of paramagnetic austenitic steel at finite temperature: Longitudinal spin fluctuations in multicomponent alloys
}

\author{
Zhihua Dong, ${ }^{1, *}$ Stephan Schönecker, ${ }^{1, \dagger}$ Dengfu Chen, ${ }^{2}$ Wei Li, ${ }^{1}$ Mujun Long, ${ }^{2}$ and Levente Vitos ${ }^{1,3,4}$ \\ ${ }^{1}$ Applied Materials Physics, Department of Materials Science and Engineering, KTH - Royal Institute of Technology, \\ SE-10044 Stockholm, Sweden \\ ${ }^{2}$ College of Materials Science and Engineering, Chongqing University, Chongqing 400030, People's Republic of China \\ ${ }^{3}$ Department of Physics and Astronomy, Division of Materials Theory, Uppsala University, Box 516, SE-75120 Uppsala, Sweden \\ ${ }^{4}$ Research Institute for Solid State Physics and Optics, Wigner Research Center for Physics, P.O. Box 49, H-1525 Budapest, Hungary
}

(Received 23 June 2017; revised manuscript received 30 October 2017; published 13 November 2017)

\begin{abstract}
We propose a first-principles framework for longitudinal spin fluctuations (LSFs) in disordered paramagnetic (PM) multicomponent alloy systems and apply it to investigate the influence of LSFs on the temperature dependence of two elastic constants of PM austenitic stainless steel Fe15Cr15Ni. The magnetic model considers individual fluctuating moments in a static PM medium with first-principles-derived LSF energetics in conjunction with describing chemical disorder and randomness of the transverse magnetic component in the single-site alloy formalism and disordered local moment (DLM) picture. A temperature-sensitive mean magnetic moment is adopted to accurately represent the LSF state in the elastic-constant calculations. We make evident that magnetic interactions between an LSF impurity and the PM medium are weak in the present steel alloy. This allows gaining accurate LSF energetics and mean magnetic moments already through a perturbation from the static DLM moments instead of a tedious self-consistent procedure. We find that LSFs systematically lower the cubic shear elastic constants $c^{\prime}$ and $c_{44}$ by $\sim 6 \mathrm{GPa}$ in the temperature interval $300-1600 \mathrm{~K}$, whereas the predominant mechanism for the softening of both elastic constants with temperature is the magneto-volume coupling due to thermal lattice expansion. We find that non-negligible local magnetic moments of $\mathrm{Cr}$ and $\mathrm{Ni}$ are thermally induced by LSFs, but they exert only a small influence on the elastic properties. The proposed framework exhibits high flexibility in accurately accounting for finite-temperature magnetism and its impact on the mechanical properties of PM multicomponent alloys.
\end{abstract}

DOI: 10.1103/PhysRevB.96.174415

\section{INTRODUCTION}

First-principles calculations in the framework of density functional theory (DFT) play an increasingly important role in the understanding and design of properties of technologically relevant materials, many of which are based on $\mathrm{Fe}, \mathrm{Co}$, or $\mathrm{Ni}$. When it comes to finite-temperature properties of these and the other magnetic transition metals and their alloys, the lack of a complete theory for itinerant electron magnetism remains one of the biggest challenges in condensed matter physics despite intensive efforts [1,2]. Since the finite-temperature properties of such materials are typically strongly coupled to their magnetic state, which in turn is intimately interrelated to the electronic states, a proper account of magnetic excitations, both transverse and longitudinal in nature, is thus of fundamental interest.

The magnetization dynamics and finite-temperature behavior of magnetic materials is often described by mapping the quantum system onto a classical model Hamiltonian for the spin system, and based on an adiabatic approximation [3,4]. The Heisenberg Hamiltonian is probably the most widespread descriptor of the magnetic energy for the orientational degrees of freedom of local-site moments, and has been explored for the $3 d$-band magnets $\mathrm{Fe}, \mathrm{Co}$, and $\mathrm{Ni}$ to investigate, among other things, low-temperature collective magnon excitations $[5,6]$ and the magnetic phase transition $[7,8]$. The validity of

\footnotetext{
*zhihuad@kth.se

†stesch@kth.se
}

the Heisenberg Hamiltonian obviously relies on the robustness of the local-moment variables with varying spin configuration and temperature.

Magnetic thermodynamics in real metallic magnets is, however, significantly more complicated and in addition characterized by fluctuations of the longitudinal magnetic component of the local moments, depending on the degree of electron localization. In $\mathrm{Fe}, \mathrm{Co}$, and $\mathrm{Ni}$, the exchange splitting is of the same order as the electronic bandwidth, making the energy scale of longitudinal spin fluctuations (LSFs) already accessible at temperatures below the magnetic phase transition [9-11]. To improve the predictions of the localized (Heisenberg) limit, one may account for LSFs in such phenomenological models, and extended model Hamiltonians have indeed been proposed in recent years $[7,8,12,13]$. On the other hand, parameters entering magnetic Hamiltonians, e.g., the exchange coupling strength, are typically kept frozen as a function of spin configuration and temperature, which introduces a dependence on the reference state in which these parameters were obtained. To overcome this drawback, several updating procedures were suggested in the literature $[3,14,15]$.

Any predictive first-principles electronic theory for the magnetic phase transition in $3 d$ metallic magnets must support the important magnetic fluctuations. Considerable progress in the first-principles formulation of the spin-fluctuation problem was achieved in notable works of Györffy et al. and Staunton et al. by merging the disordered local moment (DLM) scheme with spin DFT in a mean-field type theory $[4,16,17]$. Implemented in multiple-scattering methods combined with the coherent-potential approximation (CPA) [18,19] (formulated 
originally for the electronic structure problem of substitutional disorder) the DLM picture provides a feasible tool to describe from first principles the electronic structure in the presence of orientational spin fluctuations. One of the earliest successful predictions of this theory were wave-vector- and energydependent "local exchange splittings" for paramagnetic (PM) body-centered cubic (bcc) Fe [20], a feature that was qualitatively confirmed in spectroscopic measurements [21-23]. The neglect of dynamical effects of the spin fluctuations is an obvious shortcoming of the DLM picture, whereas the assumption on independently fluctuating local moments (neglect of magnetic short range order correlations) can be, at least in part, improved on by incorporating the effects of the Onsager cavity field into the theory [24].

Paramagnetic austenitic stainless steels, extensively used in various industrial and domestic applications, are a prominent example of magnetic metallic materials, wherein magnetic excitations at ambient temperature have been shown to produce important effects on fundamental mechanical parameters, such as the stacking fault energy and the elastic constants [25-27]. Although several other alloying elements might be deliberately added, austenitic stainless steels are essentially disordered multicomponent alloys composed of $\mathrm{Fe}$ and concentrated amounts of approximately $13-25$ at. \% $\mathrm{Cr}$ and 8-24 at. \% $\mathrm{Ni}$. In these steels, the PM state and the face-centered cubic (fcc) structure of $\gamma$-Fe, which exists only at temperatures above $1189 \mathrm{~K}$ in pure $\mathrm{Fe}$, can survive far below ambient temperature due to the presence of $\mathrm{Ni}[28,29]$.

Applying the DLM picture to PM Fe-Cr-Ni alloy with typical composition yields persistent local magnetic moment only on Fe. In the early works [25-27], temperature-driven variations of the longitudinal magnetic components in $\mathrm{Fe}-\mathrm{Cr}$ $\mathrm{Ni}$ were induced by adding a mean-field magnetic entropy term to a local magnetic moment dependent free-energy function without considering their energetics and statistics. Therefore, a more elaborate description of temperature-dependent mechanical properties in consideration of LSFs for all alloy species is highly desirable, not only for the here considered austenitic stainless steels, but for multicomponent PM alloys in general.

We recently formulated an accurate and efficient computational scheme for LSFs in the DLM state of Fe adopting a mean local magnetic moment approach to represent the energy of the spin fluctuation state [30]. The purpose of the present paper is to extend this scheme to more complicated cases, while maintaining accuracy and efficiency of the approach. In Sec. II, we formulate a methodological framework for LSFs in PM multicomponent alloy systems from first-principles spin DFT calculations. Our starting point is a model Hamiltonian for LSFs in the DLM state with parameters determined from first-principles calculations. It describes the on-site LSF energetics of individual fluctuating spins in a static PM medium and assumes the Boltzmann distribution for the longitudinal magnetic component. The energy of the spin fluctuation state is represented by mean moments. Drawing upon the single-site alloy theory formalism for chemical and magnetic randomness, we introduce two approaches that allow obtaining the LSF energetics for complex materials in a computationally more feasible way than previous studies. Following the computational details presented in Sec. III, the accuracy of the approaches is scrutinized in Sec. IV, where we apply our methodology to PM fcc Fe and austenitic stainless steel Fe15Cr15Ni. This ternary alloy exhibits the magnetic order-disorder transition at $\sim 26 \mathrm{~K}[29,31]$. We investigate the evolution of thermal spin fluctuations as a function of temperature and derive the LSF contribution to the temperature dependence of two elastic constants of this alloy steel.

\section{METHODOLOGY}

We begin by briefly outlining the general theoretical framework using the example of a unary system on a Bravais lattice (e.g., pure bcc Fe) in Sec. II A. The model is then generalized to multicomponent alloy systems in Sec. II B. Two alternative realizations of the model, i.e., the supercell representation and alloy formalism in the single-site approximation, are elucidated in Sec. II A. Next we introduce two approximations, the one shot from static equilibrium approach (OSA) at the end of Sec. II B and the fluctuating medium approximation (FMA) in Sec. II C, to improve the computational feasibility. In Sec. II D, we adopt the formulated framework to establish the thermal LSF model for the Fe-Cr-Ni ternary alloys forming the main building block of austenitic stainless steel.

We assess the aforementioned approximations in Sec. IV A by comparing results derived from different approaches: (i) supercell calculation with OSA (for variational magnetic states on neighbor sites), (ii) single-site formalism with OSA, and (iii) single-site formalism at OSA in combination with FMA. Throughout the paper we assume that all magnetic moments are in units of the Bohr magneton $\left(\mu_{\mathrm{B}}\right)$ and omit the units in the equations.

\section{A. LSF theory for PM monoatomic systems}

As magnetic variables of the PM electron system, we employ the magnetization density integrated within the Voronoi polyhedron around a lattice site $i$ and denoted by $\boldsymbol{\mu}_{i}$. We consider fluctuations of the longitudinal magnetic component $\mu_{i}$ of this local magnetic moment $\boldsymbol{\mu}_{i}=\mu_{i} \boldsymbol{e}_{i}, \boldsymbol{e}_{i}$ being its orientation and $\bar{\mu}_{i}$ its mean, and assume an adiabatic separation of fast electron dynamics from the slow spin fluctuations relevant here $[4,32]$. This site is embedded in a homogeneous PM medium created by $N-1$ other sites with randomly oriented spins, whose local magnetic moment magnitudes are fixed to their mean values $\left\{\bar{\mu}_{j}\right\}_{j \neq i}$. This notation abbreviates the set of mean moments, where $j$ runs over all sites but site $i$. A local magnetic moment $\boldsymbol{\mu}_{i}$ interacts with all other fluctuating spins via the PM medium. The energy of this state can be represented by $[33,34]$

$$
E=\sum_{i=1}^{N} E_{\mathrm{hom}}\left(\left\{\bar{\mu}_{j}\right\}\right)+\sum_{i=1}^{N} E_{\mathrm{LSF}}\left(\mu_{i} ;\left\{\bar{\mu}_{j}\right\}_{j \neq i}\right),
$$

where $E_{\text {hom }}$ is the energy of the homogeneous PM medium. The on-site term $E_{\mathrm{LSF}}$ denotes the LSF energy that measures the energy of changing the local magnetic moment at site $i$ from $\bar{\mu}_{i}$ to $\mu_{i}$. The direct interaction between two or more fluctuating lattice site magnetic moments in the PM state, for example through a bilinear form $\boldsymbol{\mu}_{i} \boldsymbol{\mu}_{j}$, are of higher order and neglected in the present model. The statistical mechanics of the LSFs is assumed to be governed by Boltzmann statistics $[7,33,35,36]$. For all $i$, the mean value $\bar{\mu}_{i}$ should 
be consistent with that of the medium it fluctuates in. This requires an iterative determination of $\left\{\bar{\mu}_{i}\right\}$, which may be a rather cumbersome procedure in practice.

For the purpose of clarification, we elucidate this model for two different realizations: (i) a supercell representation and (ii) the alloy formalism in the single-site approximation (or CPA) $[18,19]$. In the supercell picture, the LSF distribution is established for a site $i$ fluctuating in the homogeneous PM medium formed by the remaining $N-1$ sites. Since for a unary metal all sites of the Bravais lattice are equivalent, the system is described by a single LSF distribution and a single mean moment $\bar{\mu}$. $E_{\mathrm{LSF}}$ may then be mapped out by constrained spin-density functional calculations. In the present implementation, we represent the PM medium in the supercell picture by the DLM state like in the single-site approximation. Alternatively, the supercell could also be used to realize a random spin distribution (e.g., by adopting suitable special quasirandom structures).

In the single-site alloy formalism, one obtains an effective medium that possesses the symmetry properties of the underlying lattice, and the effective medium for an ideal paramagnet of totally random spins is represented by the DLM state $[4,16,17]$, which neglects mutual correlations of probability distributions of the orientations of the local moments at different lattice sites [37]. The DLM state is modeled by its alloy analogy: an equiconcentration binary alloy with collinear local magnetic moments (e.g., $\mathrm{Fe}_{0.5}^{\uparrow} \mathrm{Fe}_{0.5}^{\downarrow}$ ). Thus, a single site suffices to model the homogeneous PM medium, and $E_{\mathrm{LSF}}$ may be derived by considering the energy of a spin impurity fluctuation (IF) [38]

$$
E_{\mathrm{LSF}}(\mu)=\left.\lim _{\delta \rightarrow 0} \frac{\partial E_{\mathrm{IF}}\left(\mu^{\delta^{\prime}} ; \bar{\mu}^{1-\delta^{\prime}}\right)}{\partial \delta^{\prime}}\right|_{\delta} .
$$

The IF energy $E_{\mathrm{IF}}$ defines the energy of changing the local magnetic moment of the spin impurity $\delta$ from $\bar{\mu}$ to $\mu$. It is determined from a four-component DLM state, i.e., $\mathrm{Fe}_{\delta / 2}^{\uparrow} \mathrm{Fe}_{\delta / 2}^{\downarrow} \mathrm{Fe}_{(1-\delta) / 2}^{\uparrow} \mathrm{Fe}_{(1-\delta) / 2}^{\downarrow}$ in alloy notation, where the total concentration of the IF with magnetic moment $\mu^{\delta}$ is $\delta$ and that of the DLM effective medium $1-\delta$. Similarly to the supercell representation, the system is again described by a single mean moment $\bar{\mu}$. It should be noted that an alternative but numerically virtually identical route (in the limit of small $\delta)$ to obtain $E_{\mathrm{IF}}$ is a three-component magnetic state [38] (i.e., $\mathrm{Fe}_{\delta} \mathrm{Fe}_{(1-\delta) / 2}^{\uparrow} \mathrm{Fe}_{(1-\delta) / 2}^{\downarrow}$ ). Throughout this work we employ the DLM state for both the IF and the medium, which will be particularly important in formulating an approximation introduced in Sec. II C.

It should be noted that the homogeneous PM medium is constructed by isotropic probability distributions for the fluctuations of local moment orientations, and this construction does not involve a specific temperature $[4,16,17]$. Nevertheless, the self-consistent determination of the mean moment of the DLM effective medium allows us to associate a temperature with it, namely, the one the LSF distribution was established with through Boltzmann statistics.

It is clear that the supercell realization and the single-site approximation of the magnetic model may lead to slightly different LSFs and mean moments. Fortunately, the next level approximations based on fluctuating medium and sublattices

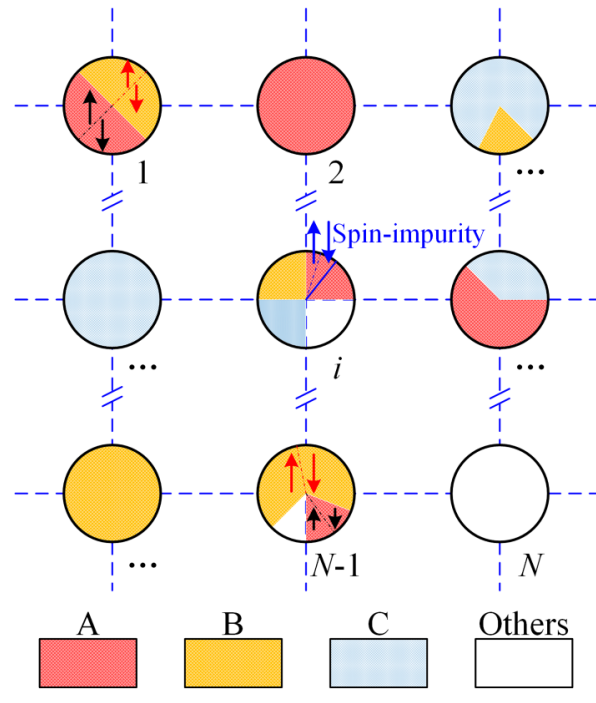

FIG. 1. Schematic of the multicomponent alloy model. The dashed lines illustrate the lattice planes and $i=1, \ldots, N$ is the site index. Different filling patterns stand for different alloy components A, B, C and any other alloy elements (illustrated by the empty site). The corresponding areas sketch the on-site concentration of different alloy species. The arrows sketch the spin-up and spin-down orientations of each alloy component (DLM state), whereas their lengths represent the local magnetic moment magnitudes, which may vary with alloy species and site. It should be noted that the spin state is only sketched for two sites but they actually may exist on all sites. A single spin impurity of species A on site $i$ is sketched.

partially resolve this difference. These approximations are described in the following sections.

We primarily draw upon the single-site alloy theory formalism in the further course. For the sake of clarification, we make contact with the supercell representation at times. In the following we proceed to multicomponent-alloy systems.

\section{B. LSF theory for PM multicomponent alloys}

Let $\mathrm{A}_{c^{\mathrm{A}}} \mathrm{B}_{c^{\mathrm{B}}} \cdots \mathrm{M}_{c^{\mathrm{M}}}$ denote the chemical composition of a multicomponent alloy consisting of components $I=$ $\mathrm{A}, \mathrm{B}, \ldots, \mathrm{M}$ with corresponding atomic concentrations $c^{I}=$ $c^{\mathrm{A}}, c^{\mathrm{B}}, \ldots, c^{\mathrm{M}}$, which are normalized as $\sum_{I} c^{I}=1$. We describe the configuration of the multicomponent alloy by a supercell containing $N$ sites; see the sketch in Fig. 1. For a site $i$, we have the alloy occupation $\mathrm{A}_{c_{i}^{\mathrm{A}}} \mathrm{B}_{c_{i}^{\mathrm{B}}} \cdots \mathrm{M}_{c_{i}^{\mathrm{M}}}$ with the concentrations satisfying $\sum_{I} c_{i}^{I}=1$. We notice that several of these site concentrations can be zero.

Before discussing the LSFs in our model, we wish to emphasize two aspects. First, ordered systems with multiple sublattices occupied by specific types of atoms may straightforwardly be reached from our model in Fig. 1 by regularly taking the concentration for a particular component to be 1 , e.g., a two-sublattice binary system for which $c_{\alpha}^{\mathrm{A}}=1$ and $c_{\beta}^{\mathrm{B}}=1$ for sublattices $\alpha$ and $\beta$, respectively. On the other hand, disordered multicomponent alloys represented within the single-site approximation are also part of this model. For instance, the $N=1$ case on a close-packed cubic lattice models a multicomponent alloy on a single site. Hence, our model naturally contains the usual supercell approach to 
alloys (e.g., the one corresponding to the special quasirandom configuration) as well as the CPA. This is an important feature since it allows one to treat, for instance, structural defects in random alloys. We should notice that the ordered case includes the monoatomic system described in Sec. II A when represented with supercells, whereas the $N=1$ case can account for the monoatomic system represented in the single-site approximation.

Because the magnetic moment may vary with site and alloy component, they are distinguished by using the site and alloy indexes, that is, $\mu_{i}^{I}$ and the corresponding mean $\bar{\mu}_{i}^{I}$. The multicomponent systems can be treated in a similar way to a monoatomic system if we omit the direct LSF couplings between different sites and alloy species, i.e., we neglect the higher-order terms in the magnetic Hamilton formalism [33]. Accordingly, the multiple-LSF picture simplifies in our model to individual spin impurities independently fluctuating in an effective medium, where all sites and alloy species (except the spin impurity) exhibit their mean magnetic moments. The indirect LSF interactions are accounted for through the individual mean moments.

In the following we establish the LSF energy for the multicomponent alloy model analogously to the monoatomic case Eq. (2). The effective medium for a spin IF $\mu_{i}^{I, \delta}$ consists of three parts. It is formed by the remaining fraction of this component on the same site $\bar{\mu}_{i}^{I, c_{i}^{I}-\delta}$, the other chemical components on the same site $\left\{\bar{\mu}_{j}^{J, c_{j}^{J}}\right\}_{j=i}^{J \neq I}$, and all remaining sites $\left\{\bar{\mu}_{j}^{J, c_{j}^{J}}\right\}_{j \neq i}$. In addition to the alloy components (indices $I, J)$, the inner upper index indicates the corresponding concentration on the sites (indices $i, j$ ). In order to simplify the notation, this complex effective medium for $\mu_{i}^{I, \delta}$ is succinctly denoted by $\langle\bar{\mu}\rangle_{i}^{I, \delta}$. Following Eq. (2), $E_{\mathrm{LSF}}$ can be evaluated by taking the derivative of the IF energy with respect to the spin-impurity concentration $\delta$,

$$
E_{\mathrm{LSF}}\left(\mu_{i}^{I}\right)=\left.\lim _{\delta \rightarrow 0} \frac{\partial E_{\mathrm{IF}}\left(\mu_{i}^{I, \delta^{\prime}} ;\langle\bar{\mu}\rangle_{i}^{I, \delta^{\prime}}\right)}{\partial \delta^{\prime}}\right|_{\delta} .
$$

Knowing $E_{\mathrm{LSF}}\left(\mu_{i}^{I}\right)$ allows determining the continuous LSF density distribution $x_{i}^{I}$ for $\mu_{i}^{I}$ using Boltzmann statistics [30,39], viz.,

$$
x_{i}^{I}\left(\mu_{i}^{I}\right)=Z^{-1}\left(\mu_{i}^{I}\right)^{2} \exp \left[\frac{-E_{\mathrm{LSF}}\left(\mu_{i}^{I}\right)}{k_{\mathrm{B}} T}\right],
$$

with partition function

$$
Z=\int\left(\mu_{i}^{I}\right)^{2} \exp \left[\frac{-E_{\mathrm{LSF}}\left(\mu_{i}^{I}\right)}{k_{\mathrm{B}} T}\right] d \mu_{i}^{I},
$$

where $T$ is the temperature and $k_{\mathrm{B}}$ the Boltzmann constant. The LSF density distribution can be used to derive the mean moment $\bar{\mu}_{i}^{I}$.

The spin-impurity concentration dependent $E_{\mathrm{IF}}$ is straightforwardly extracted from the total energy $E_{\text {tot }}$ between the configuration including a finite concentration of IFs relative to the spin-impurity-free effective medium,

$$
E_{\mathrm{IF}}\left(\mu_{i}^{I, \delta} ;\langle\bar{\mu}\rangle_{i}^{I, \delta}\right)=E_{\mathrm{tot}}\left(\mu_{i}^{I, \delta} ;\langle\bar{\mu}\rangle_{i}^{I, \delta}\right)-E_{\mathrm{tot}}\left(\left\{\bar{\mu}_{j}^{J}\right\}\right) .
$$

Substituting Eq. (6) into Eq. (3) and acknowledging that $E_{\mathrm{LSF}}$ is evaluated by a small but finite concentration change in practice, we arrive at

$$
E_{\mathrm{LSF}}\left(\mu_{i}^{I}\right) \approx \frac{E_{\mathrm{tot}}\left(\mu_{i}^{I, \delta} ;\langle\bar{\mu}\rangle_{i}^{I, \delta}\right)-E_{\mathrm{tot}}\left(\left\{\bar{\mu}_{j}^{J}\right\}\right)}{\delta} .
$$

Although not necessary to formulate the theory, our alloy model allows introducing the concept of sublattices. By definition, all sites belonging to a sublattice $\alpha$ possess identical chemical, structural, and LSF properties. In the context of modeling LSF in a magnetically and chemically totally disordered state by the DLM scheme in combination with the CPA, the introduction of sublattices deserves, however, an additional comment. Describing for example PM bcc Fe by the primitive or conventional unit cell leads to identical results only if LSFs simultaneously occur on all sites making up the unit cell (as distinct magnetic states lower the symmetry). Invariance with respect to the choice of the unit cell can be achieved by organizing these sites in a single sublattice and including IFs on all sites of this sublattice. All above equations retain their form apart from replacing the site index by the sublattice index, while also ensuring normalization of $E_{\mathrm{LSF}}$ to a single site. In Sec. IV A, we enlarge on the numerical differences of deriving $E_{\mathrm{LSF}}$ from the primitive unit cell and from embedding a fluctuating site in a supercell.

Reaching the self-consistency requirement on $\left\{\bar{\mu}_{i}^{I}\right\}$ mentioned above becomes prohibitively cumbersome as the number of sites or alloy components grows. In practice, we thus abandon the iterative determination of the mean moments and introduce simplifications to make the inclusion of LSFs in the calculation of materials properties feasible. The simplest remedy may be referred to as the one shot from static equilibrium approach (OSA) and considers thermal LSFs around the static PM equilibrium; i.e., the LSF distribution for a component $I$ is established by fixing those local magnetic moments to their static equilibrium values that form the effective medium. The static equilibrium values are derived through a separate unconstrained spin-density functional calculation neglecting longitudinal magnetic excitations. Denoting the magnitude of a static equilibrium local magnetic moment by $\mu_{j, 0}^{J}$, and the static medium for an IF $\mu_{i}^{I, \delta}$ by $\langle\mu\rangle_{i, 0}^{I, \delta}, E_{\mathrm{LSF}}$ in the OSA is approximated by

$$
E_{\mathrm{LSF}}^{\mathrm{OSA}}\left(\mu_{i}^{I}\right) \approx \frac{E_{\mathrm{tot}}\left(\mu_{i}^{I, \delta} ;\langle\mu\rangle_{i, 0}^{I, \delta}\right)-E_{\mathrm{tot}}\left(\left\{\mu_{j, 0}^{J}\right\}\right)}{\delta} .
$$

LSF distributions derived from this approach or the iterative way are not expected to differ significantly in the case of a weak coupling to the effective medium. In Sec. IV A we show that this is indeed the case for the present alloy system.

\section{Fluctuating medium approximation}

The LSF energy $E_{\mathrm{LSF}}\left(\mu_{i}^{I}\right)$ in the single-site approximation and derived spin fluctuation properties are invariant under the choice of the spin-impurity concentration, if the total energy $E_{\text {tot }}$ on the right-hand side of Eq. (7) is linear in the spinimpurity concentration for all $\mu_{i}^{I}$. Motivated by the results of our calculations for PM fcc Fe presented in Sec. IV A, where we show that $E_{\mathrm{LSF}}\left(\mu_{i}^{I}\right)$ weakly deviates from linearity in $\delta$ for $\mu_{i}^{I} \leqslant 2.5 \mu_{\mathrm{B}}$, we introduce the fluctuating medium 
approximation (FMA) to the on-site LSF energy,

$$
E_{\mathrm{LSF}}^{\mathrm{FMA}}\left(\mu_{i}^{I}\right) \approx \frac{E_{\mathrm{tot}}\left(\mu_{i}^{I, c_{i}^{I}} ;\langle\bar{\mu}\rangle_{i}^{I, c_{i}^{I}}\right)-E_{\mathrm{tot}}\left(\left\{\bar{\mu}_{j}^{J}\right\}\right)}{c_{i}^{I}},
$$

by maximizing $\delta$ to the concentration of component $I$ at site $i$, i.e., $c_{i}^{I}$. An increasing $\delta$ may be interpreted as an excitation of increasingly more spins in the PM medium, whose longitudinal components vary synchronously. Simultaneously, the fraction of isospecies magnetic moments forming part of the effective medium disappears (i.e., the term $\bar{\mu}_{i}^{I, c_{i}^{l}-\delta}$ in the single-site approximation). In the special case of a monoatomic system on a Bravais lattice, the FMA uses the energy of the entire, homogeneously fluctuating PM medium to establish the onsite LSF distribution. An insignificant dependence of $E_{\mathrm{LSF}}\left(\mu_{i}^{I}\right)$ on $\delta$ corresponds to the limit of weak indirect interactions and results in LSF distributions that do not strongly depend on the choice of $\delta$ in $\left[0, c_{i}^{I}\right]$. The FMA can be used in combination with the OSA, Eq. (8); in fact, this combination was employed in recent investigations of the impact of LSFs on the elastic constants and the thermal expansion of PM fcc and bcc Fe $[30,40]$. In Sec. IV A, we briefly investigate the numerical differences of deriving $E_{\mathrm{LSF}}$ through an IF Eq. (8) and through the FMA.

\section{LSFs in austenitic stainless steel}

The present PM austenitic stainless steel $\mathrm{Fe}-\mathrm{Cr}-\mathrm{Ni}$ is modeled as a random solid solution in the fcc structure using the primitive unit cell, i.e., $N=1$ and $I, J=\mathrm{Fe}, \mathrm{Cr}$, or $\mathrm{Ni}$. $c^{\mathrm{Fe}}=0.70, c^{\mathrm{Cr}}=0.15$, and $c^{\mathrm{Ni}}=0.15$ denote the atomic concentrations of $\mathrm{Fe}, \mathrm{Cr}$, and $\mathrm{Ni}$, respectively, The site index is dropped henceforth.

Adopting the FMA and the OSA, the LSF energy for Fe reads

$$
E_{\mathrm{LSF}}^{\mathrm{FMA}+\mathrm{OSA}}\left(\mu^{\mathrm{Fe}}\right) \approx \frac{E_{\mathrm{tot}}\left(\mu^{\mathrm{Fe}} ; \mu_{0}^{\mathrm{Cr}}, \mu_{0}^{\mathrm{Ni}}\right)-E_{\mathrm{tot}}\left(\left\{\mu_{0}^{J}\right\}\right)}{c^{\mathrm{Fe}}} .
$$

In order to determine the LSF distribution, the total energy was computed for a six-component DLM alloy, i.e., $\mathrm{Fe}_{c^{\mathrm{Fe}} / 2}^{\uparrow \mu^{\mathrm{Fe}}} \mathrm{Fe}_{c^{\mathrm{Fe}} / 2}^{\downarrow \mu^{\mathrm{Fe}}}$. $\mathrm{Cr}_{c^{c \mathrm{r}} / 2}^{\uparrow \mu_{0}^{\mathrm{Cr}}} \mathrm{Cr}_{c^{\mathrm{Cr}} / 2}^{\downarrow \mu_{0}^{\mathrm{Cr}}} \cdot \mathrm{Ni}_{c^{\mathrm{Ni}} / 2}^{\uparrow \mu_{0}^{\mathrm{Ni}}} \mathrm{Ni}_{c^{\mathrm{Ni}} / 2}^{\downarrow \mu^{\mathrm{Ni}}}$, with variable magnetic moment $\mu^{\mathrm{Fe}}$ for $\mathrm{Fe}$, and constrained magnetic moments $\mu_{0}^{\mathrm{Cr}}$ and $\mu_{0}^{\mathrm{Ni}}$ for $\mathrm{Cr}$ and $\mathrm{Ni}$ atoms, respectively. Similar expressions to Eq. (10) and calculational schemes apply to LSFs on $\mathrm{Cr}$ and $\mathrm{Ni}$.

In the numerical calculation, the local magnetic moment range for $\mathrm{Fe}$ was chosen from 0 to $3.0 \mu_{\mathrm{B}}$ in intervals of $0.5 \mu_{\mathrm{B}}$, whereas the ranges were 0 to $2.5 \mu_{\mathrm{B}}$ and 0 to $2.0 \mu_{\mathrm{B}}$ for $\mathrm{Cr}$ and $\mathrm{Ni}$, respectively, with identical intervals. To obtain the continuous LSF distributions, we fitted an eighth-order Landau expression $E_{\mathrm{LSF}}-E_{\mathrm{LSF}}(\mu=0)=a \mu^{2}+b \mu^{4}+c \mu^{6}+d \mu^{8}$ to the energies calculated on each discrete mesh. Accordingly, the LSF distributions of $\mathrm{Fe}, \mathrm{Cr}$, and $\mathrm{Ni}$ are established based on a series of constrained spin-density functional calculations. To ease the comparison between different species, in Sec. IV we plot $E_{\mathrm{LSF}}$ with respect to $E_{\mathrm{LSF}}(\mu=0)$.

In the following we elaborate on how to consider LSFs and their distributions $x^{I}$ in the calculation of the elastic properties of the present ternary alloy. Upon binning each probability density distribution $x^{I}$ for $\mu^{I}$ into probabilities $x_{1}^{I}$,
$x_{2}^{I}, \ldots, x_{n}^{I}, \sum_{k} x_{k}^{I}=1$, for discrete local magnetic moments $\mu_{1}^{I}, \mu_{2}^{I}, \ldots, \mu_{n}^{I}$, the PM state of the austenitic stainless steel in the presence of LSFs may be modeled by the multicomponent DLM picture, viz.,

$$
\begin{aligned}
& \left(\mathrm{Fe}_{x_{1}^{\mathrm{e}} / 2}^{\uparrow \mu_{1}} \mathrm{Fe}_{x_{1}^{\mathrm{Fe}} / 2}^{\downarrow \mu_{1}} \ldots \mathrm{Fe}_{x_{n}^{\mathrm{fe}} / 2}^{\uparrow \mu_{n}} \mathrm{Fe}_{x_{n}^{\mathrm{Fe}} / 2}^{\downarrow \mu_{n}}\right)_{c_{\mathrm{Fe}}} . \\
& \left(\mathrm{Cr}_{x_{1}^{\mathrm{x}_{1}} / 2}^{\uparrow \mu_{1}} \mathrm{Cr}_{x_{1}^{\mathrm{Cr}} / 2}^{\downarrow \mu_{1}} \ldots \mathrm{Cr}_{x_{n}^{\mathrm{r}} / 2}^{\uparrow \mu_{n}} \mathrm{Cr}_{x_{n}^{\mathrm{Cr}} / 2}^{\downarrow \mu_{n}}\right)_{c^{\mathrm{Cr}}} \text {. } \\
& \left(\mathrm{Ni}_{x_{1}^{\mathrm{Ni}} / 2}^{\uparrow \mu_{1}} \mathrm{Ni}_{x_{1}^{\mathrm{Ni}} / 2}^{\downarrow \mu_{1}} \ldots \mathrm{Ni}_{x_{n}^{\mathrm{Ni}} / 2}^{\uparrow \mu_{n}} \mathrm{Ni}_{x_{n}^{\mathrm{N}} / 2}^{\downarrow \mu_{n}}\right)_{c^{\mathrm{Ni}}},
\end{aligned}
$$

where each probability $x_{i}^{I}$ can be directly related to an atomic fraction, which has to be normalized by the corresponding chemical concentration in the present ternary random alloy. In general, distinct numbers of bins $n$ and local magnetic moment intervals may be chosen for the alloy components.

Aiming to reduce the computational load of modeling the multicomponent DLM picture Eq. (11), we proposed an alternative approach [30] to represent the continuous LSF distributions by a single, mean magnetic moment for each species denoted by $m_{\mathrm{sf}}^{I}$. Accordingly, $m_{\mathrm{sf}}^{I}$ was defined by

$$
m_{\mathrm{sf}}^{I}=\sqrt{\int \mu^{2} x^{I}(\mu) d \mu} .
$$

This mean moment was shown to accurately represent the energy of the LSF state in the description of thermomechanical properties such as the elastic constants in PM Fe [30], and we investigate its performance for the $\mathrm{Fe}-\mathrm{Cr}-\mathrm{Ni}$ system in Sec. IV C. In the present investigation, the upper limit of the integral in Eq. (12) was limited to 3.5, 3.0, and $2.5 \mu_{\mathrm{B}}$ for Fe, $\mathrm{Cr}$, and $\mathrm{Ni}$, respectively; i.e., for all components we used the Landau expression to extrapolate by $0.5 \mu_{\mathrm{B}}$ beyond the upper limit of the chosen discrete magnetic moment mesh. In place of the multicomponent DLM picture, in this work we employ $m_{\text {sf }}^{I}$ to model the impact of LSFs on the elastic constants in the PM Fe-Cr-Ni system, and Eq. (11) simplifies to the following six-component alloy,

$$
\mathrm{Fe}_{c^{\mathrm{Fe}} / 2}^{\uparrow m_{\mathrm{sf}}^{\mathrm{Fe}}} \mathrm{Fe}_{c^{\mathrm{Fe}} / 2}^{\downarrow m_{\mathrm{sf}}^{\mathrm{Fe}}} \cdot \mathrm{Cr}_{c^{\mathrm{Cr}} / 2}^{\uparrow m_{\mathrm{sf}}^{\mathrm{Cr}}} \mathrm{Cr}_{c^{\mathrm{Cr}} / 2}^{\downarrow m_{\mathrm{sf}}^{\mathrm{Cr}}} \cdot \mathrm{Ni}_{c^{\mathrm{Ni}} / 2}^{\uparrow m_{\mathrm{si}}^{\mathrm{Ni}}} \mathrm{Ni}_{c^{\mathrm{Ni}} / 2}^{\downarrow m_{\mathrm{sf}}^{\mathrm{Ni}}}
$$

\section{TOTAL ENERGY SCHEME, LATTICE EXPANSION, AND ELASTIC PROPERTIES}

The ab initio calculations were performed in the framework of density functional theory (DFT) [41]. The scalar-relativistic one-electron Kohn-Sham equations in combination with the local-density approximation (LDA) [42] were solved with the exact muffin-tin orbitals method [43-46] adopting the soft-core scheme. The magnetic and chemical disorders in PM Fe-Cr-Ni were described by the DLM picture $[4,16,17]$ in combination with the CPA $[18,19]$. The total energy was calculated applying the full charge-density technique [47] based on the quasi-nonuniform gradient-level exchangecorrelation approximation (QNA) [48-50]. The QNA has been shown to predict highly accurate equations of state for bcc and fcc Fe [30].

Being a mean-field theory, magnetic and chemical shortrange order effects are not accounted for in our calculations. Considering the relatively low magnetic transition temperature $(\sim 26 \mathrm{~K})$ for the present fcc alloy [29,31], the magnetic 
short-range order is expected to be weak at the temperatures considered here (300-1600 K). Based on the parametrization carried out within the ordered magnetic background, the chemical short-range order in $\mathrm{Fe}-\mathrm{Cr}-\mathrm{Ni}$ alloys was demonstrated to be non-negligible [51]. Nevertheless, as shown in the above work, the short-range order in the fcc Fe-Cr-Ni alloys is weaker than in the bcc alloys, which have very high order-disorder transition temperature and exhibit short-range order even at temperatures close to melting temperature [51]. In addition, taking the disordered magnetism in the PM state into consideration, a weak short-range order has been reported for PM fcc $\mathrm{Fe} 21 \mathrm{Cr} 23 \mathrm{Ni}$ [52], in reasonable agreement with the existing experimental data. Thus the proper account of magnetism at finite temperature was pointed out to be crucial for the theoretical description of the austenitic stainless steels. Concerning the impact of the chemical short-range order on the mechanical properties, to our best knowledge no theoretical study has been reported so far for Fe-based alloys. It was, however, demonstrated that chemical short-range order gives a negligible contribution to the elastic properties of nonmagnetic binary alloys [53]. Since here we concentrate on the thermal LSFs in PM fcc multicomponent alloys and its impacts on the elastic property, the chemical short-range order effects are omitted.

The equilibrium Wigner-Seitz radius $(w)$ of the PM Fe15Cr15Ni austenitic stainless steel was determined from an exponential Morse-type function [54] fitted to $a b$ initio total energies. The theoretical Wigner-Seitz radii at finite temperatures were determined by rescaling the equilibrium radius using the experimental linear thermal expansion coefficient $20.77 \times 10^{-6} \mathrm{~K}^{-1}$, which was in fact determined by dilatometry for the commercial austenitic stainless steel Fe18Cr8Ni at elevated temperatures [55]. The small deviation in the alloy composition is, however, expected to yield a small effect on the thermal lattice expansion coefficient of Fe15Cr15Ni. According to the calculations, the theoretical static equilibrium Wigner-Seitz radius of PM fcc Fe15Cr15Ni amounts to 2.6119 bohrs, and at $300 \mathrm{~K}$ the lattice expands to 2.6282 bohrs. This latter result is comparable to the experimental observation for $\mathrm{Fe} 15 \mathrm{Cr} 15 \mathrm{Ni}$ at $300 \mathrm{~K} w_{\text {Expt. }}=$ 2.6479 bohrs [56] with a deviation of approximately $-0.74 \%$, showing relatively high accuracy.

Volume-conserving orthorhombic and monoclinic deformations were adopted in the calculation of the two cubic shear elastic constants $c^{\prime}$ and $c_{44}$ [43]. In addition to the LSF calculations, elastic constants were also calculated using the conventional floating-spin (FS) scheme for comparison, where longitudinal magnetic excitations were neglected. The Brillouin zone sampling was done using uniformly distributed $k$ points with density that ensures the necessary numerical accuracy for the elastic-constant calculations.

\section{RESULTS AND DISCUSSION}

\section{A. LSF interactions among different atomic sites and alloy species}

The purpose of this section is to look into the various approaches to obtain $E_{\mathrm{LSF}}$ introduced in Sec. II and to learn about indirect local magnetic moment interactions in the single-site alloy theory formalism. We focus on pure PM fcc
Fe first and investigate LSFs set up for two choices of the unit cell, i.e., the primitive unit cell and a supercell with a single embedded fluctuating site. Second, for Fe15Cr15Ni and using the FMA, we scrutinize how LSFs on each alloy component are affected by the magnetic state of the medium defined by the moments of the other two alloy components. The main quantity of interest is the mean moment defined in Eq. (12) and its stability is used as a qualitative measure of the interaction strength with the medium.

\section{PMfcc Fe}

As mentioned above, LSFs for PM fcc Fe are investigated for two choices of the unit cell. On the one hand, a supercell representation was chosen to describe the situation of a single fluctuating site embedded in a PM medium. To this end, calculations were performed for a $2 \times 2 \times 2$ supercell in terms of the conventional fcc unit cell, where all 32 sites were modeled by the DLM state. $E_{\mathrm{LSF}}$ was mapped out by changing the local magnetic moment magnitude of the fluctuating site from 0 to $2.5 \mu_{\mathrm{B}}$ with an interval of $0.5 \mu_{\mathrm{B}}$, whereas the moments $\mu^{\mathrm{NB}}$ of the remaining 31 sites were fixed (NB stands for neighbors). In order to estimate the interaction strength between the fluctuating site with its surrounding medium and the impact on $E_{\mathrm{LSF}}$, we compare $\mu^{\mathrm{NB}}=0 \mu_{\mathrm{B}}$ with $\mu^{\mathrm{NB}}=1.84 \mu_{\mathrm{B}} \equiv \mu_{0,1400 \mathrm{~K}}^{\mathrm{Fe}}$. The former value for $\mu^{\mathrm{NB}}$ corresponds to the nonmagnetic state and the latter value is the static DLM moment of fcc Fe at the Wigner-Seitz radius $w_{1400 \mathrm{~K}}^{\mathrm{Fe}}=2.6951$ bohrs, which was previously derived by means of the experimental thermal expansion coefficient of PM fcc Fe in combination with the QNA equilibrium volume [30]. On the other hand, we drew upon the primitive fcc unit cell and compare the IF formalism combined with the OSA [Eq. (8)] to the FMA [Eq. (9)]. In all cases, the results were obtained at $T=1400 \mathrm{~K}$ and corresponding $w_{1400 \mathrm{~K}}^{\mathrm{Fe}}$.

Figure 2 shows the results for $E_{\mathrm{LSF}}$ for the above four cases. First, we compare the results from the supercell calculations. In response to an increase of $\mu^{\mathrm{NB}}$ from 0 to $1.84 \mu_{\mathrm{B}}$, small differences in the LSF energies are obtained (the largest difference occurs at $2.5 \mu_{\mathrm{B}}$ ), and the derived mean magnetic moment increases slightly from 2.07 to $2.08 \mu_{\mathrm{B}}$. The stabilities of the LSF energy and mean magnetic moment indicate that the magnetic states of the fluctuating site and the surrounding medium interact weakly. Deriving $E_{\mathrm{LSF}}$ from the IF formalism combined with the OSA for the static magnetic moment $\mu_{0,1400 \mathrm{~K}}^{\mathrm{Fe}}$ yields an energy curve virtually identical to the one from the supercell representation with $\mu^{\mathrm{NB}}=1.84 \mu_{\mathrm{B}}$; see Fig. 2. The corresponding mean magnetic moment $2.09 \mu_{\mathrm{B}}$ from the IF formalism is very close as well. Thus, assuming the same magnitude of the local magnetic moments in the effective medium, the embedded single fluctuating site and the IF formalism give consistent results, indicating that the two pictures align. It should be noted in Fig. 2 that the supercell curve for $\mu^{\mathrm{NB}}=1.84 \mu_{\mathrm{B}}$ and the FMA curve are not identical at $\mu=1.84 \mu_{\mathrm{B}}$ since the reference energies at $\mu=0 \mu_{\mathrm{B}}$ differ.

The LSF energy through the FMA has a similar shape to the three other functions, but possesses a slightly deeper energy minimum and raises more rapidly beyond the minimum; see Fig. 2. This leads to a somewhat smaller mean magnetic 


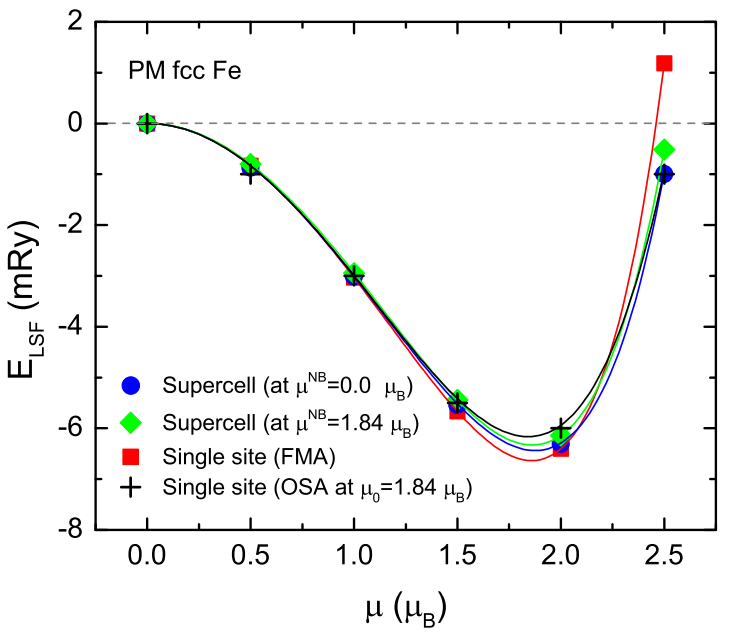

FIG. 2. On-site LSF energy $E_{\mathrm{LSF}}$ of PM fcc Fe as a function of the spin impurity magnetic moment $\mu$ evaluated with alternative approaches: a single fluctuating spin embedded in a supercell with two distinct magnetic moments on neighboring sites (i.e., $\mu^{\mathrm{NB}}=$ 0 or $1.84 \mu_{\mathrm{B}}$ ), the IF formalism combined with the OSA (static local magnetic moment $1.84 \mu_{\mathrm{B}}$ ), and the FMA. All calculations were performed at the Wigner-Seitz radius $w_{1400 \mathrm{~K}}=2.6951$ bohrs.

moment $2.01 \mu_{\mathrm{B}}$. All in all, the four mean magnetic moment values are consistent and spread apart by less than 5\%, which provides support for the the consistency of these four approaches and the accuracy of the FMA in the case of PM fcc Fe.

\section{PMfcc FeCrNi}

In the following part, we investigate more closely how LSFs in $\mathrm{Fe} 15 \mathrm{Cr} 15 \mathrm{Ni}$ depend on the magnetic state of the effective medium adopting the FMA. All results were obtained for $T=$ $1600 \mathrm{~K}$ at the Wigner-Seitz radius $w_{1600 \mathrm{~K}}=2.6987$ bohrs.

Figure 3 shows elaborately how the mean magnetic moment of either $\mathrm{Fe}, \mathrm{Cr}$, or $\mathrm{Ni}$ atoms vary with the magnetic state of the effective medium defined by the local magnetic moments of the other two alloy components. Before focusing on the details, it is worth noting an essential general trait of the coupling. The functional dependencies $m_{\mathrm{sf}}(\mu)$ are quadratic in $\mu$ unless $\mu$ becomes large, and a change in $\mu$ on the order of $1-2 \mu_{\mathrm{B}}$ induces a relatively small change in $m_{\mathrm{sf}}$ on the order of $0.1 \mu_{\mathrm{B}}$. This is an obvious signature of the weak coupling in this system.

In more detail, for the mean magnetic moment of iron $m_{\text {sf }}^{\mathrm{Fe}}$ shown in Fig. 3(a), small changes are observed when the magnetic moments of $\mathrm{Cr} \mu^{\mathrm{Cr}}$ and $\mathrm{Ni} \mu^{\mathrm{Ni}}$ are varied. Specifically, increasing $\mu^{\mathrm{Cr}}$ from 0 to $1.5 \mu_{\mathrm{B}}$ with $\mu^{\mathrm{Ni}}=0 \mu_{\mathrm{B}}$ fixed results in an almost stable $m_{\mathrm{sf}}^{\mathrm{Fe}}$ at $\sim 2.05 \mu_{\mathrm{B}}$ with very small positive slope versus $\mu^{\mathrm{Cr}}$. There is little change in the trend of $m_{\mathrm{sf}}^{\mathrm{Fe}}$ except a shift by $-0.02 \mu_{\mathrm{B}}$ when $\mu^{\mathrm{Ni}}$ is increased from 0 to $1.0 \mu_{\mathrm{B}}$. As indicated by the green arrow, this drop of $m_{\mathrm{sf}}^{\mathrm{Fe}}$ for $\mu^{\mathrm{Cr}}=0 \mu_{\mathrm{B}}$ can also be obtained from the curve labeled $\mu^{\mathrm{Ni}}$ (at $\mu^{\mathrm{Cr}}=0 \mu_{\mathrm{B}}$ ) (red solid bullets). On the other hand, increasing $\mu^{\mathrm{Ni}}$ from 0 to $1.0 \mu_{\mathrm{B}}$ with $\mu^{\mathrm{Cr}}=0 \mu_{\mathrm{B}}$ fixed slightly declines $m_{\mathrm{sf}}^{\mathrm{Fe}}$ by $\sim 0.02 \mu_{\mathrm{B}}$, a trend that is insignificantly altered by increasing $\mu^{\mathrm{Cr}}$ to $1.5 \mu_{\mathrm{B}}$.

Similarly weak LSF interactions are found in the determination of $m_{\mathrm{sf}}^{\mathrm{Cr}}$ and $m_{\mathrm{sf}}^{\mathrm{Ni}}$. As indicated by the red solid bullets
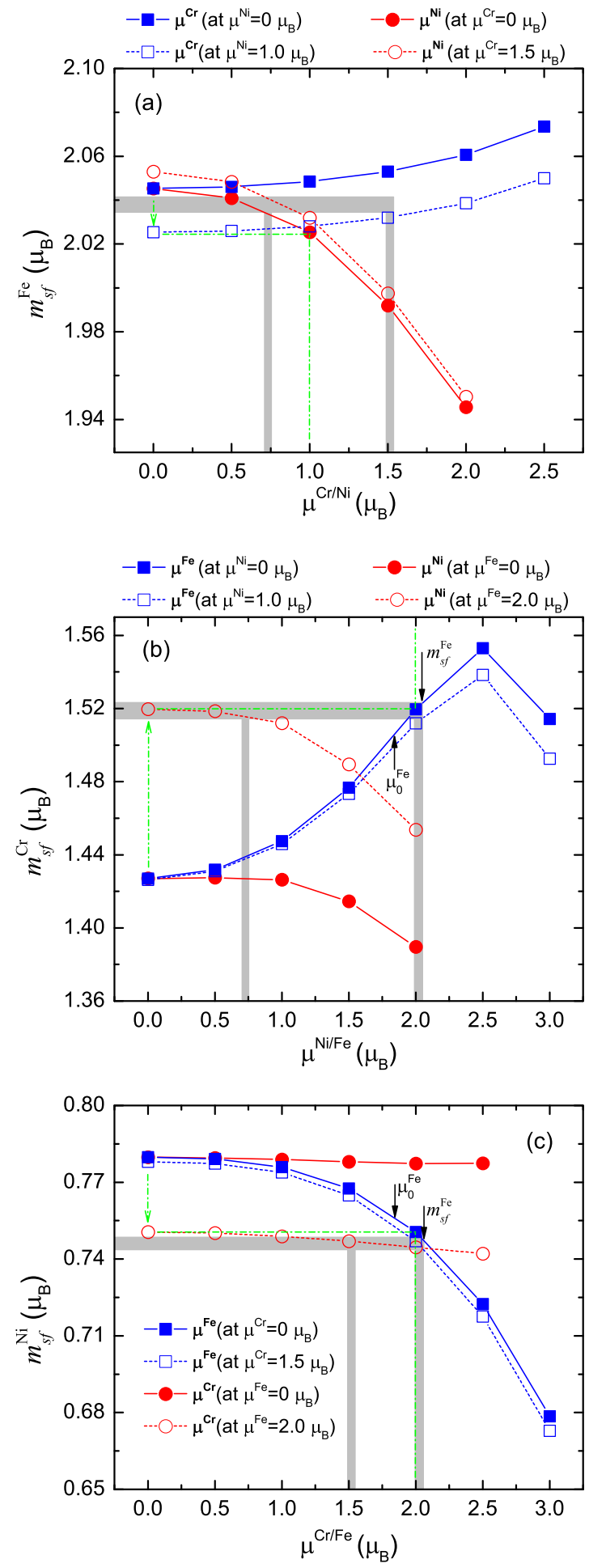

FIG. 3. Mean magnetic moment $m_{\mathrm{sf}}^{I}$ of alloy component $I$ in PM $\mathrm{Fe} 15 \mathrm{Cr} 15 \mathrm{Ni}$ as a function of the local magnetic moments of the other two alloy species in the effective medium, where $I$ is $\mathrm{Fe}, \mathrm{Cr}$, and $\mathrm{Ni}$ for panels (a), (b), and (c), respectively. While the magnetic moment of one species in the medium is constrained to the value in parentheses, the magnetic moment of the other one is varied. The shaded horizontal and vertical bars indicate the self-consistent solution, the black arrows indicate the static magnetic moment and the OSA mean magnetic moment for Fe, whereas the green arrows are explained in the text. All calculations were performed for $1600 \mathrm{~K}$ at the Wigner-Seitz radius $w_{1600 \mathrm{~K}}=2.6987$ bohrs. 
TABLE I. Static DLM local magnetic moments $\mu_{0,1600 \mathrm{~K}}$, selfconsistent mean magnetic moments $\bar{m}_{\text {sf }}$ (uncertainty $\pm 0.005 \mu_{\mathrm{B}}$ ), and OSA mean magnetic moments $m_{\mathrm{sf}}$ (relative error with respect to the former in parentheses) for $\mathrm{Fe}, \mathrm{Cr}$, and $\mathrm{Ni}$ in $\mathrm{Fe} 15 \mathrm{Cr} 15 \mathrm{Ni}$ random alloy evaluated at $T=1600 \mathrm{~K}$ and the Wigner-Seitz radius $w_{1600 \mathrm{~K}}=$ 2.6987 bohrs. All moments are in units of $\mu_{\mathrm{B}}$.

\begin{tabular}{lccc}
\hline \hline & \multicolumn{3}{c}{ Site } \\
\cline { 2 - 4 } Moment & $\mathrm{Fe}$ & $\mathrm{Cr}$ & $\mathrm{Ni}$ \\
\hline$\mu_{0,1600 \mathrm{~K}}$ & 1.840 & 0.000 & 0.000 \\
$\bar{m}_{\mathrm{sf}}$ & 2.038 & 1.519 & 0.746 \\
$m_{\mathrm{sf}}$ & $2.045(0.3 \%)$ & $1.505(-0.9 \%)$ & $0.757(1.5 \%)$ \\
\hline \hline
\end{tabular}

in Fig. 3(b), $m_{\mathrm{sf}}^{\mathrm{Cr}}$ exhibits little variation with increasing $\mu^{\mathrm{Ni}}$ from 0 to $1.0 \mu_{\mathrm{B}}$ at $\mu^{\mathrm{Fe}}=0 \mu_{\mathrm{B}}$. This is also the case for $m_{\mathrm{sf}}^{\mathrm{Ni}}$ shown in Fig. 3(c); i.e., a small variation of $m_{\mathrm{sf}}^{\mathrm{Ni}}$ is observed by increasing $\mu^{\mathrm{Cr}}$ from 0 to $1.5 \mu_{\mathrm{B}}$. As indicated by the green arrows in Figs. 3(b) and 3(c), the $m_{\mathrm{sf}}^{\mathrm{Cr}}$ and $m_{\mathrm{sf}}^{\mathrm{Ni}}$ curves are nearly rigidly shifted by $0.09 \mu_{\mathrm{B}}$ and $-0.03 \mu_{\mathrm{B}}$, respectively, when $\mu^{\mathrm{Fe}}$ in the medium increases from 0 to $2.0 \mu_{\mathrm{B}}$. Furthermore, as indicated by the negligible differences between the blue solid square data and blue open square data in Figs. 3(b) and $3(\mathrm{c})$, the effect of $\mu^{\mathrm{Fe}}$ on $m_{\mathrm{sf}}^{\mathrm{Cr}}$ and $m_{\mathrm{sf}}^{\mathrm{Ni}}$ is rather insensitive to a variation of $\mu^{\mathrm{Ni}}$ and $\mu^{\mathrm{Cr}}$, respectively.

In order to evaluate the performance of the OSA, we investigate the proximity of the OSA mean moments to the self-consistent mean magnetic moments $\bar{m}_{\text {sf }}$. The data shown in Fig. 3 allow determining the latter within an uncertainty of $\pm 0.005 \mu_{\mathrm{B}}$, which is sufficient for the present purpose. Self-consistency is achieved when the three mean magnetic moments (ordinates) are consistent with the values of the medium they fluctuate in (i.e., for each alloy component, the moment of the second element along the abscissa combined with the two curves for the third element). The self-consistent graphical solution is highlighted in Figs. 3(a)-3(c) by the shaded horizontal and vertical bars, where the width of the horizontal bars $\left(0.01 \mu_{\mathrm{B}}\right)$ indicates the error bar for $\bar{m}_{\mathrm{sf}}$.

The self-consistent mean magnetic moments, the OSA mean magnetic moments $\left(m_{\mathrm{sf}}\right)$, and the static DLM local magnetic moments $\left(\mu_{0,1600 \mathrm{~K}}\right)$, from which the OSA moments were derived, are compared in Table I. The $\bar{m}_{\text {sf }}$ differ significantly from the static DLM local magnetic moments, whereas all three OSA mean magnetic moments are surprisingly close to their self-consistent value. The largest deviation (1.5\%) is observed for Ni. It should be noted that the somewhat larger magnitude of the errors for $\mathrm{Cr}$ and $\mathrm{Ni}$ can be explained by their lower chemical concentrations.

In summary, the calculations adopting the FMA showed that the OSA yields accurate mean magnetic moments due to a weak coupling between the LSFs and the effective medium they fluctuate in. Thus, the use of the OSA is justified for the following investigations for $\mathrm{Fe} 15 \mathrm{Cr} 15 \mathrm{Ni}$.

\section{B. Evolution of magnetism of PM austenitic stainless steel with temperature}

We present the detailed analysis of the LSF energies and derived density distributions for PM Fe15Cr15Ni at $1600 \mathrm{~K}$

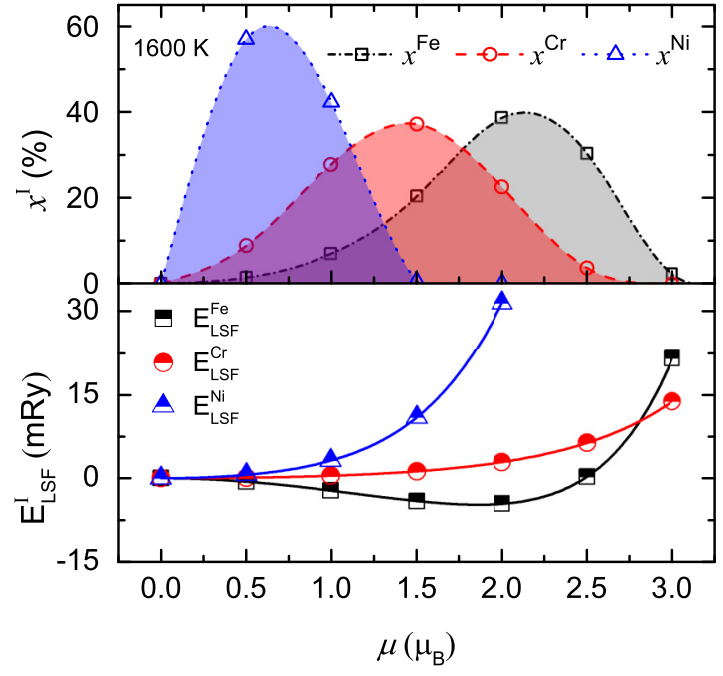

FIG. 4. On-site LSF energies $E_{\mathrm{LSF}}^{I}$ as a function of the local magnetic moment $\mu$ for LSFs on $\mathrm{Fe}, \mathrm{Cr}$, or Ni sites (lower panel) and the corresponding LSF density distributions at $1600 \mathrm{~K}$ (upper panel) in PM Fe15Cr15Ni. $E_{\mathrm{LSF}}^{I}$ was calculated for each alloy component $I$ adopting the FMA. All calculations were performed at the same Wigner-Seitz radius $w_{1600 \mathrm{~K}}=2.6987$ bohrs. The solid lines in the lower panel are the fitted polynomial Landau expansions to the data (points) and were used to establish the continuous LSF distributions as sketched by the transparent areas.

in Fig. 4. These results were obtained with the methodology elaborated in Sec. II D and include the thermal lattice expansion; i.e., all calculations were performed at the Wigner-Seitz radius $w_{1600 \mathrm{~K}}=2.6987$ bohrs. The corresponding static local magnetic moments are given in Table I.

As shown in Fig. 4, the LSF energy for Fe exhibits a shallow minimum at the static local magnetic moment $1.84 \mu_{\mathrm{B}}$, whereas $E_{\mathrm{LSF}}^{I}$ monotonically increases for $\mathrm{Cr}$ and $\mathrm{Ni}$ consistent with their vanishing static local magnetic moments. The high temperature induces fluctuations of the longitudinal magnetic component resulting in LSF distributions with different widths and centers of gravity. The density distributions for both $\mathrm{Cr}$ and $\mathrm{Ni}$ are rather symmetric and peak at significantly large local magnetic moment magnitudes, but the particularly shallow LSF energy curve of $\mathrm{Cr}$ makes more intense magnetic excitations possible and leads to a larger mean value. $x^{\mathrm{Fe}}$ has a clear negative skew and concentrates around the spontaneous magnetic moment $\mu_{0,1600 \mathrm{~K}}^{\mathrm{Fe}}$.

In order to characterize the density distributions at $1600 \mathrm{~K}$ and for temperatures below, the mean magnetic moment according to Eq. (12) is shown as a function of temperature in Fig. 5 for each alloy component in PM Fe15Cr15Ni. The means were evaluated for LSF distributions taking into account the thermal expansion. For comparison, the static magnetic moments $\mu_{0}^{I}$ derived from the FS calculations are also shown, where the temperature dependence merely arises from the magneto-volume coupling.

As is evident from Fig. 5, LSFs are responsible for the persisting finite mean local magnetic moments for $\mathrm{Cr}$ and $\mathrm{Ni}$ in the entire considered temperature interval, as $\mu_{0}^{\mathrm{Cr}}$ and $\mu_{0}^{\mathrm{Ni}}$ are identically zero throughout. Cr exhibits a larger $m_{\mathrm{sf}}^{I}$ due to energetically more preferable excitations rooted in the 


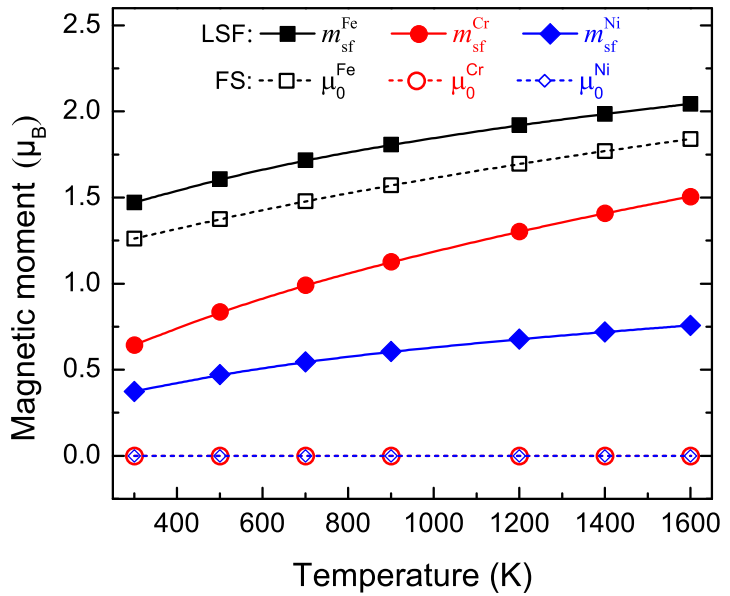

FIG. 5. Temperature dependence of the mean local magnetic moment $m_{\mathrm{sf}}^{I}$ and the static local magnetic moment $\mu_{0}^{I}$ for $\mathrm{Fe}, \mathrm{Cr}$, or $\mathrm{Ni}$ in $\mathrm{PM} \mathrm{Fe} 15 \mathrm{Cr} 15 \mathrm{Ni}$ taking into account thermal expansion.

flatter energy curve (Fig. 4). With increasing the temperature from 300 to $1600 \mathrm{~K}$, the mean magnetic moments of $\mathrm{Cr}$ and $\mathrm{Ni}$ gradually increase from 0.64 and 0.37 to $1.50 \mu_{\mathrm{B}}$ and $0.76 \mu_{\mathrm{B}}$, respectively. As regards Fe, increasing local magnetic moments are obtained in the FS calculations. Taking LSFs into account, the magnetic moment of Fe is elevated by $\sim 0.2 \mu_{\mathrm{B}}$ with little change in its temperature dependence. When the temperature increases from 300 to $1600 \mathrm{~K}, m_{\mathrm{sf}}^{\mathrm{Fe}}$ gradually increases from 1.47 to $2.04 \mu_{\mathrm{B}}$.

\section{Temperature-dependent elastic constants of PM austenitic stainless steel}

The temperature-dependent elastic properties of PM $\mathrm{Fe} 15 \mathrm{Cr} 15 \mathrm{Ni}$ and the impact of LSFs are quantitatively estimated in the following. Before presenting the results below, we verified the flexibility of the mean magnetic moment Eq. (12) to represent the LSF distributions in the elastic-constant calculations for PM austenitic stainless steel, which was previously done only for pure PM Fe [30]. To this end, we compare the two elastic constants $\left(c^{\prime}\right.$ and $\left.c_{44}\right)$ calculated through the multicomponent DLM picture for the LSF distributions with the results from the mean magnetic moment approach. In the former case, the thermal LSFs of the ternary alloy were modeled as a 30-component alloy [Eq. (11)] by using the determined LSF density distributions of each alloy species, whereas the magnetic state was modeled as a 6-component alloy adopting the mean magnetic moments derived from the same LSF density distributions [Eq. (13)]. All the calculations were carried out at the fixed Wigner-Seitz radius of 2.6499 bohrs with varying the temperature from 300 to $1600 \mathrm{~K}$. With respect to the results derived from the LSF distributions, $c^{\prime}$ and $c_{44}$ of PM Fe15Cr15Ni through the mean magnetic moment scheme are slightly overestimated and underestimated by less than $2.9 \%$ and $1.4 \%$, respectively, in the whole temperature range. Thus, the mean magnetic moment scheme yields accurate elastic constants for $\mathrm{Fe} 15 \mathrm{Cr} 15 \mathrm{Ni}$ and was applied in the following elastic-property calculations.

We recall that the effect of temperature enters by means of the magneto-volume coupling due to the thermal expansion

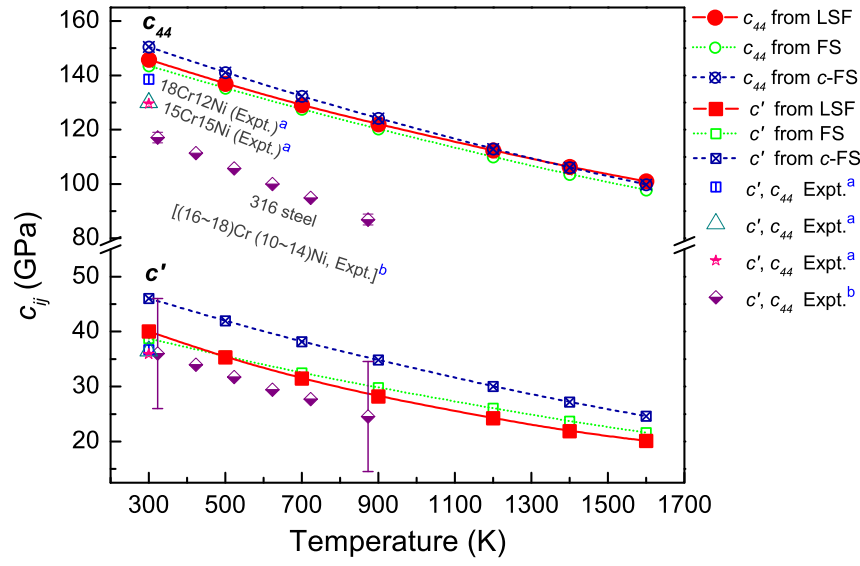

FIG. 6. Temperature dependence of the single-crystal elastic constants $c^{\prime}$ and $c_{44}$ of PM Fe15Cr15Ni calculated with different schemes. In addition to the LSF results, elastic constants adopting the FS and $c$-FS schemes along with available experimental data from (a) Ref. [56] and (b) Ref. [57] are presented for comparison. It should be noted that the error bars indicated for two experimental points in each case apply to all data from (b) Ref. [57].

and the evolution of the LSFs with temperature represented by the mean magnetic moments shown in Fig. 5. The calculated elastic constants $c^{\prime}$ and $c_{44}$ of PM Fe $15 \mathrm{Cr} 15 \mathrm{Ni}$ in consideration of LSFs are shown in Fig. 6 along with the available experimental data for comparison. We can see that the elastic constants of PM austenitic stainless steel predicted by the proposed formalism exhibit relatively high accuracy with respect to the measurements. Particularly, at room temperature, the predicted $c^{\prime}$ and $c_{44}$ are close to the measurements for $\mathrm{Fe} 15 \mathrm{Cr} 15 \mathrm{Ni}$ and $\mathrm{Fe} 18 \mathrm{Cr} 12 \mathrm{Ni}$ from Ref. [56]. The average deviations between theoretical and experimental results are $\sim 3.5 \mathrm{GPa}(8.8 \%)$ and $\sim 12 \mathrm{GPa}(8.2 \%)$ for $c^{\prime}$ and $c_{44}$, respectively. Compared to the commercial austenitic stainless steel 316L [57], similar thermal softenings of $c^{\prime}$ and $c_{44}$ are predicted. The temperature coefficients are presented in Table II and are discussed below. The calculated $c^{\prime}$ of $\mathrm{PM}$ Fe $15 \mathrm{Cr} 15 \mathrm{Ni}$ is relatively close to that of $316 \mathrm{~L}$ with a small positive deviation of approximately $4 \mathrm{GPa}$, whereas the calculated $c_{44}$ is clearly larger compared to the $316 \mathrm{~L}$ steel. However, it should be noted that the $c_{44}$ reported in Ref. [57] lies approximately $10 \mathrm{GPa}$ below the observed values from Ref. [56].

For comparison, both elastic constants of PM Fe $15 \mathrm{Cr} 15 \mathrm{Ni}$ were calculated employing two alternative schemes. In addition to the LSF results calculated from $m_{\mathrm{sf}}^{I}$, we performed conventional FS and constrained FS $(c$-FS) calculations. In the $c$-FS calculations, the magnetic moments of $\mathrm{Fe}, \mathrm{Cr}$, and $\mathrm{Ni}$ were fixed to the static values $\mu_{0}^{I}$ (shown in Fig. 5) without allowing for relaxation of their magnitudes upon lattice distortion employed to determine the particular elastic constant. The difference between the FS and $c$-FS results can help us to estimate the size of the error associated with constraining the magnitudes of the mean magnetic moments in the elastic-constant calculations. As shown in Fig. 6, $c$-FS consistently yields larger elastic constants than FS, and the deviations between FS and $c$-FS schemes are less than $\sim 6 \mathrm{GPa}$ for both $c^{\prime}$ and $c_{44}$. Using this information, one can define 
TABLE II. Temperature coefficients of the elastic constants for PM Fe15Cr15Ni. The temperature-coefficient intervals derived from the LSF $\left(C_{\mathrm{LSF}}\right)$ and $c$-FS $\left(C_{c-\mathrm{FS}}\right)$ calculations are given at the considered lower $(300 \mathrm{~K})$ and upper $(1600 \mathrm{~K})$ temperature limits. Available experimental data from Refs. [56,57] and previous theoretical predictions from Ref. [27] are collected in $C_{\text {ref }}$ for comparison, where the reference temperature is specified in parentheses. All temperature coefficients are given in units of $10^{-2} \mathrm{GPa} \mathrm{K}^{-1}$.

\begin{tabular}{lcrr}
\hline \hline & $C_{\mathrm{LSF}}$ & $C_{c-\mathrm{FS}}$ & $C_{\text {ref }}$ \\
\hline$c^{\prime}$ & $-2.5 \sim-0.7$ & $-2.1 \sim-1.2$ & $-1.9(300 \mathrm{~K}$, Calc.) [27] \\
& & & $-2.1(300 \mathrm{~K}$, Expt.) [56] \\
& & & a $-2.2 \sim-1.9(323-873 \mathrm{~K}$, Expt.) [57] \\
$c_{44}$ & $-4.6 \sim-2.2$ & $-4.8 \sim-3.0$ & $-4.0(300 \mathrm{~K}$, Calc.) [27] \\
& & & $-4.9(300 \mathrm{~K}$, Expt.) [56] \\
& & & $-5.1(323-873 \mathrm{~K}$, Expt.) [57] \\
\hline \hline
\end{tabular}

${ }^{\mathrm{a}}$ The temperature coefficients were derived for commercial austenitic stainless steel 316L.

a correction (of similar magnitude and negative sign) to the LSF results in order to eliminate the shift of the elastic constants towards higher values arising from the constrained mean moments.

The effect of LSFs on both elastic constants can be directly quantified from the difference between the $c$-FS and LSF results. Taking spin fluctuations into account, the predicted $c^{\prime}$ lowers by $\sim 6 \mathrm{GPa}$ in the whole temperature range, whereas $c_{44}$ declines by $\sim 5 \mathrm{GPa}$ at the lowest considered temperature, but the effect of LSFs gradually diminishes towards higher temperatures. Overall, the consideration of LSFs yields a better agreement with the available experiments. The different contributions from LSFs to $c^{\prime}$ and $c_{44}$ can be understood by different magnetoelastic couplings in Fe and Fe-based alloys as discussed in Refs. [27,30].

To quantify the temperature dependencies of the elastic constants in consideration of LSFs, we fitted parabolas to the predicted results shown in Fig. 6. The best fits were obtained with the following expressions:

$$
\begin{gathered}
c^{\prime}(T)=6.82 \times 10^{-6} T^{2}-2.87 \times 10^{-2} T+48.42, \\
c_{44}(T)=9.12 \times 10^{-6} T^{2}-5.17 \times 10^{-2} T+160.30,
\end{gathered}
$$

where the elastic constants are in GPa and the temperature in $\mathrm{K}$.

The temperature coefficients $C$ are then directly related to the first derivative of the fitting functions. Table II shows the temperature coefficients of $c^{\prime}$ and $c_{44}$ for PM Fe15Cr15Ni calculated from the LSF $\left(C_{\mathrm{LSF}}\right)$ and $c$-FS $\left(C_{c \text {-FS }}\right)$ schemes. Obviously, the derivatives are temperature dependent and thus in Table II we give the intervals for the coefficients corresponding to 300-1600 K. Available data from experiments and other theoretical predictions along with the coefficients derived from fitting the experimental temperature dependencies [56,57] are also listed under $C_{\text {ref }}$ for comparison. Our computed temperature coefficients $C_{\mathrm{LSF}}$ and $C_{c \text {-FS }}$ are in close agreement with the measurements [56,57] and the previous calculation [27]. $c_{44}$ shows a pronounced softening with temperature and the magnitude of its temperature coefficient clearly exceeds that of $c^{\prime}$. Compared to the $c$-FS results, which solely account for the magneto-volume coupling due to thermal lattice expansion, the absolute values of the temperature coefficients for $c_{44}$ and $c^{\prime}(T>700 \mathrm{~K})$ slightly reduce by considering LSFs. In other words, the softening of both elastic constants with temperature is mainly determined by lattice expansion and only in parts balanced by the contribution due to LSFs, which is similar to the result of Dong et al.'s study for PM Fe [30].

From the disclosed effects of LSFs on the elastic constants (approximately $6 \mathrm{GPa}$ ) and their temperature coefficients (at most $0.8 \times 10^{-2} \mathrm{GPa} \mathrm{K}^{-1}$ ) mentioned above, one may reason that the sizable local magnetic moments of $\mathrm{Cr}$ and $\mathrm{Ni}$ induced by LSFs exert only a minor influence on the elastic properties of PM Fe15Cr15Ni. In order to investigate this, additional elastic-constant calculations were carried out adopting varying local magnetic moments for $\mathrm{Cr}$ and $\mathrm{Ni}$. Specifically, $c^{\prime}$ and $c_{44}$ of PM Fe15Cr15Ni were determined as a function of the local magnetic moment of $\mathrm{Cr}$ or $\mathrm{Ni}$ with the moment of the other component constrained to zero. These calculations were performed at $700 \mathrm{~K}$ for the Wigner-Seitz radius $w_{700 \mathrm{~K}}=$ 2.6499 bohrs, and the local magnetic moment of Fe was fixed to the mean magnetic moment $m_{\mathrm{sf}}^{\mathrm{Fe}}=1.717 \mu_{\mathrm{B}}$.

We can infer from the results in Fig. 7 that the elastic properties of PM Fe15Cr15Ni are weakly dependent on the local magnetic moment of $\mathrm{Cr}$ or $\mathrm{Ni}$. Compared to the results calculated with vanishing $\mathrm{Ni}$ magnetic moment, i.e., $\mu^{\mathrm{Ni}}=$ $0 \mu_{\mathrm{B}}$, an increase to $1.0 \mu_{\mathrm{B}}$ is accompanied by a $\sim 2.2 \mathrm{GPa}$ decrease of $c^{\prime}$ and a $\sim 1.4 \mathrm{GPa}$ increase of $c_{44}$. When $\mu^{\mathrm{Cr}}$ is increased from 0 to $1.5 \mu_{\mathrm{B}}, c^{\prime}$ and $c_{44}$ slightly increase by $\sim 0.5$ and $\sim 2.7 \mathrm{GPa}$, respectively. That is, the sizable magnetic

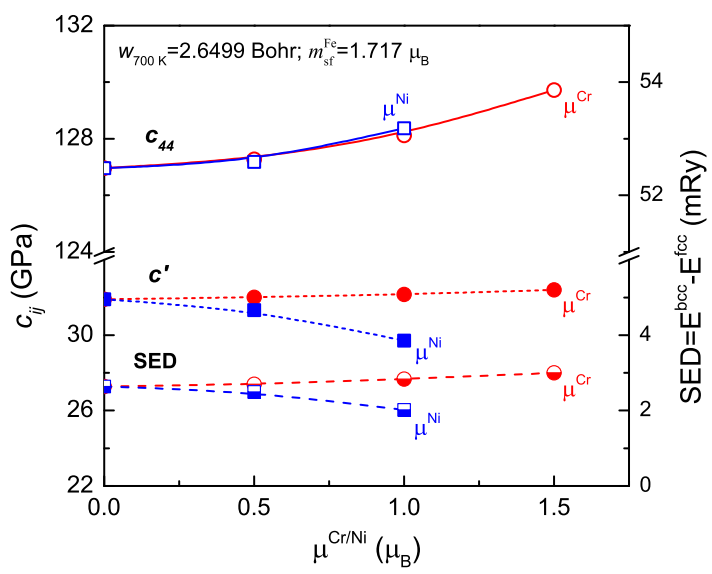

FIG. 7. $c^{\prime}, c_{44}$, and the SED for PM Fe $15 \mathrm{Cr} 15 \mathrm{Ni}$ as a function of the local magnetic moment $\mu^{\mathrm{Cr}}$ or $\mu^{\mathrm{Ni}}$ with the moment of the other component constrained to zero. The local magnetic moment of $\mathrm{Fe}$ is fixed to $m_{\mathrm{sf}}^{\mathrm{Fe}}=1.717 \mu_{\mathrm{B}}$ in all cases. The calculations were performed at $700 \mathrm{~K}$ for the Wigner-Seitz radius $w_{700 \mathrm{~K}}=2.6499$ bohrs. 
moments of $\mathrm{Cr}$ and $\mathrm{Ni}$ induced by LSFs indeed exert only a small influence on the elastic properties of the presently investigated austenitic stainless steel.

Finally, we briefly discuss the connection between the trends of the elastic constants and the structural energy difference as previously introduced in several works $[58,59]$. In particular, the tetragonal shear elastic constant $c^{\prime}$ has been demonstrated to scale with the structural energy difference (SED) between bcc and fcc structures, i.e., $\mathrm{SED}=E^{\mathrm{bcc}}-$ $E^{\mathrm{fcc}}$, for both nonmagnetic transition metals [58] and PM Fe-based alloys [59]. Namely, elements or alloys for which band-filling arguments dictate large SED have large $c^{\prime}$ as well. In the present study, the SED was calculated as a function of the local magnetic moment of $\mathrm{Cr}$ or $\mathrm{Ni}$ for $\mathrm{PM} \mathrm{Fe} 15 \mathrm{Cr} 15 \mathrm{Ni}$. As shown in Fig. 7, the dependencies of the SED on the magnetic moments of $\mathrm{Cr}$ or Ni correlate closely with the evolution of $c^{\prime}$. That is, similar scaling arguments hold for the variation of the local magnetic moment in PM austenitic stainless steels.

\section{CONCLUSIONS}

We have presented a general framework for DFT calculations of paramagnetic multicomponent alloy systems including longitudinal spin fluctuations (LSFs) and investigated the impact of these fluctuations on the elastic properties of $\mathrm{Fe} 15 \mathrm{Cr} 15 \mathrm{Ni}$ austenitic stainless steel at finite temperature. The underlying model for LSFs considers the on-site energetics $E_{\mathrm{LSF}}$ of an individual fluctuating moment in a static paramagnetic medium and assumes a classical probability distribution for the longitudinal magnetic component. The complete information for the system may be obtained by establishing $E_{\mathrm{LSF}}$ for each distinct site, e.g., by constrained spin-density functional calculations. To ensure that the mean value of each fluctuating site is consistent with that of the paramagnetic medium it fluctuates in, an iterative procedure is in principle required. Drawing upon the single-site alloy theory formalism for both chemical and magnetic randomness, we have proposed and scrutinized two simplifications to obtain $E_{\mathrm{LSF}}$ : (1) the one shot from static equilibrium approach (OSA) with the aim to overcome the cumbersome iterative determination of fluctuating moments and medium, and (2) the fluctuating medium approximation (FMA) proposed to extract $E_{\mathrm{LSF}}$ from the energetics of the paramagnetic medium rather than an impurity fluctuation. LSF distributions in the present calculations have been considered through the mean magnetic moment approach scrutinized previously.

The presented methodology has been employed to shed light on the strength of indirect local magnetic moment interactions in paramagnetic fcc $\mathrm{Fe}$ and $\mathrm{Fe} 15 \mathrm{Cr} 15 \mathrm{Ni}$ by means of the exact muffin-tin orbitals total-energy method in combination with the coherent-potential approximation and disordered local moment picture. The simulations for $\mathrm{Fe}$ have yielded consistent $E_{\mathrm{LSF}}$ and mean magnetic moments for four alternative approaches (a single fluctuating spin embedded in a supercell with two distinct magnetic moments on neighboring sites, an impurity fluctuation through the OSA, and the FMA). The calculations for $\mathrm{Fe} 15 \mathrm{Cr} 15 \mathrm{Ni}$ adopting the FMA have revealed that the OSA yields mean magnetic moments in close agreement to the self-consistent values. This is an important finding as it demonstrates that the demanding iterative determination of fluctuating moments and medium can be avoided. Both sets of results signal a generally weak coupling strength between the LSFs and the effective medium defined by the rest of the alloy species in the present fcc-Fe-based materials.

The influence of LSFs on the temperature dependence of the single-crystal elastic constants $c^{\prime}$ and $c_{44}$ of PM Fe15Cr15Ni have been quantified at finite temperature. Although sizable local magnetic moments for $\mathrm{Cr}$ and $\mathrm{Ni}$ are thermally induced by LSFs, these fluctuations result in comparatively small effects on the temperature dependence of elastic properties of PM austenitic stainless steel. Taking LSFs into consideration, both $c^{\prime}$ and $c_{44}$ lower by $\sim 6 \mathrm{GPa}$ in the whole studied temperature range. The softening of both elastic constants with temperature is, however, mainly determined by lattice expansion (magneto-volume coupling) and only partially balanced by the contribution due to LSFs.

We consider the present work an important step towards the routine and efficient inclusion of LSFs in the DFT description of thermomechanical and thermodynamic properties of complex materials. Future studies are in progress, which focus on the role of LSFs in the finite-temperature energetics of structural defects.

\section{ACKNOWLEDGMENTS}

This work was supported by the Swedish Research Council, the Swedish Foundation for Strategic Research, the Swedish Foundation for International Cooperation in Research and Higher Education, the Chinese Scholarship Council, the Hungarian Scientific Research Fund (OTKA 109570), the Carl Tryggers Foundation, and the National Natural Science Foundation of China (NSFC; Projects No. 5151101344, No. 51374260, and No. 51504048). The calculations were performed on resources provided by the Swedish National Infrastructure for Computing (SNIC) at the National Supercomputer Centre (NSC) in Linköping.
[1] T. Moriya, Spin Fluctuations in Itinerant Electron Magnetism (Springer, Berlin, 1985).

[2] K. Baberschke, M. Donath, and W. Nolting (eds.), in BandFerromagnetism: Ground-State and Finite-Temperature Phenomena, Lecture Notes in Physics Vol. 580 (Springer, Berlin, 2001).
[3] V. P. Antropov, M. I. Katsnelson, B. N. Harmon, M. van Schilfgaarde, and D. Kusnezov, Phys. Rev. B 54, 1019 (1996).

[4] B. L. Gyốrffy, A. J. Pindor, J. Staunton, G. M. Stocks, and H. Winter, J. Phys. F 15, 1337 (1985).

[5] S. V. Halilov, H. Eschrig, A. Y. Perlov, and P. M. Oppeneer, Phys. Rev. B 58, 293 (1998). 
[6] I. Turek, J. Kudrnovský, V. Drchal, and P. Bruno, Philos. Mag. 86, 1713 (2006).

[7] N. M. Rosengaard and B. Johansson, Phys. Rev. B 55, 14975 (1997).

[8] P. W. Ma and S. L. Dudarev, Phys. Rev. B 86, 054416 (2012).

[9] F. Dietermann, L. M. Sandratskii, and M. Fähnle, J. Magn. Magn. Mater. 324, 2693 (2012).

[10] J. Kübler, Theory of Itinerant Electron Magnetism, revised edition (Oxford University Press, Oxford, 2009).

[11] V. A. Gubanov, A. I. Liechtenstein, and A. V. Postnikov, Magnetism and the Electronic Structure of Crystals (Springer, Berlin, 1992).

[12] M. Ležaić, P. Mavropoulos, J. Enkovaara, G. Bihlmayer, and S. Blügel, Phys. Rev. Lett. 97, 026404 (2006).

[13] M. Y. Lavrentiev, J. S. Wrobel, D. Nguyen-Manh, and S. L. Dudarev, Phys. Chem. Chem. Phys. 16, 16049 (2014).

[14] D. Böttcher, A. Ernst, and J. Henk, J. Magn. Magn. Mater. 324, 610 (2012).

[15] F. Pan, J. Chico, A. Delin, A. Bergman, and L. Bergqvist, Phys. Rev. B 95, 184432 (2017).

[16] A. J. Pindor, J. Staunton, G. M. Stocks, and H. Winter, J. Phys. F 13, 979 (1983).

[17] J. Staunton, B. Győrffy, A. Pindor, G. Stocks, and H. Winter, J. Magn. Magn. Mater. 45, 15 (1984).

[18] P. Soven, Phys. Rev. 156, 809 (1967).

[19] B. L. Győrffy, Phys. Rev. B 5, 2382 (1972).

[20] J. Staunton, B. L. Győrffy, A. J. Pindor, G. M. Stocks, and H. Winter, J. Phys. F 15, 1387 (1985).

[21] J. Kirschner, M. Glöbl, V. Dose, and H. Scheidt, Phys. Rev. Lett. 53, 612 (1984).

[22] E. Kisker, K. Schröder, M. Campagna, and W. Gudat, Phys. Rev. Lett. 52, 2285 (1984).

[23] E. Kisker, K. Schröder, W. Gudat, and M. Campagna, Phys. Rev. B 31, 329 (1985).

[24] J. B. Staunton and B. L. Győrffy, Phys. Rev. Lett. 69, 371 (1992).

[25] L. Vitos, P. A. Korzhavyi, and B. Johansson, Phys. Rev. Lett. 96, 117210 (2006).

[26] L. Vitos, J. O. Nilsson, and B. Johansson, Acta Mater. 54, 3821 (2006).

[27] L. Vitos and B. Johansson, Phys. Rev. B 79, 024415 (2009).

[28] L. Vitos, P. A. Korzhavyi, and B. Johansson, Nat. Mater. 2, 25 (2003).

[29] A. K. Majumdar and P. V. Blanckenhagen, Phys. Rev. B 29, 4079 (1984).

[30] Z. Dong, W. Li, S. Schönecker, S. Lu, D. Chen, and L. Vitos, Phys. Rev. B 92, 224420 (2015).

[31] Y. Ishikawa, Y. Endoh, and T. Takimoto, J. Phys. Chem. Solids 31, 1225 (1970).

[32] P. Fulde, Electron Correlations in Molecules and Solids, 3rd ed., Solid-State Sciences (Springer, Berlin, 2002).

[33] S. Shallcross, A. E. Kissavos, V. Meded, and A. V. Ruban, Phys. Rev. B 72, 104437 (2005).

[34] A. V. Ruban, S. Khmelevskyi, P. Mohn, and B. Johansson, Phys. Rev. B 75, 054402 (2007).
[35] M. Uhl, L. M. Sandratskii, and J. Kübler, Phys. Rev. B 50, 291 (1994).

[36] M. Uhl and J. Kübler, Phys. Rev. Lett. 77, 334 (1996).

[37] I. Turek, V. Drchal, J. Kudrnovský, M. Šob, and P. Weinberger, Electronic Structure of Disordered Alloys, Surfaces, and Interfaces (Springer US, Boston, MA, 1997).

[38] A. V. Ruban and H. L. Skriver, Phys. Rev. B 55, 8801 (1997).

[39] L. M. Sandratskii, Phys. Rev. B 78, 094425 (2008).

[40] Z. Dong, W. Li, D. Chen, S. Schönecker, M. Long, and L. Vitos, Phys. Rev. B 95, 054426 (2017).

[41] P. Hohenberg and W. Kohn, Phys. Rev. 136, B864 (1964).

[42] W. Kohn and L. J. Sham, Phys. Rev. 140, A1133 (1965).

[43] L. Vitos, Computational Quantum Mechanics for Materials Engineers: The EMTO Method and Applications, Engineering Materials and Processes Series (Springer, London, 2007), p. 235.

[44] L. Vitos, Phys. Rev. B 64, 014107 (2001).

[45] K. Kádas, L. Vitos, B. Johansson, and J. Kollár, Phys. Rev. B 75, 035132 (2007)

[46] L. Vitos, H. L. Skriver, B. Johansson, and J. Kollár, Comput. Mater. Sci. 18, 24 (2000).

[47] L. Vitos, I. A. Abrikosov, and B. Johansson, Phys. Rev. Lett. 87, 156401 (2001).

[48] H. Levämäki, M. P. J. Punkkinen, K. Kokko, and L. Vitos, Phys. Rev. B 86, 201104 (2012).

[49] H. Levämäki, M. P. J. Punkkinen, K. Kokko, and L. Vitos, Phys. Rev. B 89, 115107 (2014).

[50] L.-Y. Tian, H. Levämäki, M. Ropo, K. Kokko, A. Nagy, and L. Vitos, Phys. Rev. Lett. 117, 066401 (2016).

[51] J. S. Wróbel, D. Nguyen-Manh, M. Y. Lavrentiev, M. Muzyk, and S. L. Dudarev, Phys. Rev. B 91, 024108 (2015).

[52] A. V. Ruban and M. Dehghani, Phys. Rev. B 94, 104111 (2016).

[53] M. Hoffmann, A. Marmodoro, E. Nurmi, K. Kokko, L. Vitos, A. Ernst, and W. Hergert, Phys. Rev. B 86, 094106 (2012).

[54] V. L. Moruzzi, J. F. Janak, and K. Schwarz, Phys. Rev. B 37, 790 (1988).

[55] We performed the dilatometric examination for commercial austenitic stainless steel $\mathrm{Fe} 18 \mathrm{Cr} 8$ by using a DIL 402C highresolution dilatometer. The experiment was carried out at a temperature range from 300 to $1273 \mathrm{~K}$ and the relative change of sample length $(d L / L)$ was recorded online. The thermal expansion coefficient was then associated with the slope of $d L / L$ versus temperature and an average was made for the whole temperature range.

[56] A. Teklu, H. Ledbetter, S. Kim, L. A. Boatner, M. McGuire, and V. Keppens, Metall. Mater. Trans. A 35, 3149 (2004).

[57] M. R. Daymond and P. J. Bouchard, Metall. Mater. Trans. A 37, 1863 (2006).

[58] P. Söderlind, O. Eriksson, J. M. Wills, and A. M. Boring, Phys. Rev. B 48, 5844 (1993).

[59] H. Zhang, M. P. J. Punkkinen, B. Johansson, and L. Vitos, Phys. Rev. B 85, 054107 (2012). 